\title{
Circuit
}

Musiques contemporaines

\section{"L'évolution historique " et ses marges : éléments pour une lecture critique de Cinquante ans de modernité musicale de Deliège}

\section{"Historic Evolution" and its Boundaries: Elements for a Critical Reading of Célestin Deliège's Cinquante ans de modernité musicale}

\section{Béatrice Ramaut-Chevassus}

Volume 16, numéro 1, 2005

Écrire l'histoire de la musique du $\mathrm{XX}^{\mathrm{e}}$ siècle

URI : https://id.erudit.org/iderudit/902384ar

DOI : https://doi.org/10.7202/902384ar

Aller au sommaire du numéro

Éditeur(s)

Les Presses de l'Université de Montréal

ISSN

1183-1693 (imprimé)

1488-9692 (numérique)

Découvrir la revue

Citer cet article

Ramaut-Chevassus, B. (2005). « L'évolution historique » et ses marges :

éléments pour une lecture critique de Cinquante ans de modernité musicale de Deliège. Circuit, 16(1), 65-72. https://doi.org/10.7202/902384ar
Résumé de l'article

Célestin Deliège, en témoin de son temps, livre un monument, une source première, une histoire des grammaires où se croisent aussi esthétique et politique. Le concept central d'évolution historique se rapporte à la " recherche musicale ", à la « recherche sur le code ", aux « efforts de recherche de langage ". Écrire l'histoire revient alors à établir un " recensement de théories intentionnelles, prescriptives, et de poétiques nouvelles ». Les marges quant à elles évoquent la glose musicologique mais surtout les nombreux compositeurs placés en marge de cette évolution historique, de ce trajet fléché, de Darmstadt à l'Ircam. Cette dichotomie reflète donc celle que C. Deliège pose lui-même. L'article essaie de montrer qu'il existe une diversité plus grande de forces en présence dans la modernité musicale de la seconde moitié du siècle dernier. 


\title{
"L'évolution historique" et ses
} marges : éléments pour une lecture critique de Cinquante ans de modernité musicale de Deliège

\author{
par Béatrice Ramaut-Chevassus
}

La présence de Célestin Deliège dans le champ théorique est, on le sait, constante depuis le début des années 1980. Les entretiens avec Pierre Boulez, Par volonté et par hasard, publiés en 1975, en constituaient, en amont, le geste inaugural et donnaient un signe sous lequel le reste de l'œuvre s'est placé. Ce geste inaugural était la revendication d'une attention première portée à la modernité, résolument. Si Cinquante ans de modernité musicale est impressionnant, c'est qu'il s'agit du travail d'un solitaire - plus de 1000 pages pour une seule plume - monographie hors normes par ses dimensions et son poids, travail qui défie le temps morcelé et hâtif puisqu'il résulte d'une préoccupation qui a su être constante durant près de 15 ans (1988-2002) peut-on lire à la toute fin, mais sans doute plus encore d'une préoccupation qui a été celle de toute une vie. Aucun des livres de Célestin Deliège, dont le précédent, Invention musicale et idéologies publié en 1986, ne lui est en cela comparable.

Célestin Deliège donne à lire un texte dont un des aspects est aussi d'être une sorte de reportage. C'est à cause de ce récit fondé sur une histoire vécue et non sur la consultation d'archives que des moments du récit prennent un relief particulier. On lit par exemple avec intérêt le chapitre consacré au "rôle initiateur et militant de René Leibowitz", dans la diffusion en France, c'est-à-dire à Paris, de la pensée d'Arnold Schoenberg. Célestin Deliège y ajoute en effet une évocation précise de la contribution de Jean-Paul Sartre en analysant la préface à L'artiste et sa conscience - ouvrage de Leibowitz publié en 1950 - y voyant "le signe en France de la naissance de la sociologie de la musique" (p. 52). La confrontation des textes contribue aussi, en remettant en scène tous les acteurs du Paris de l'époque, à 
écrire une histoire où se croisent très efficacement esthétique, politique - avec la question de l'engagement de l'artiste -, par-delà les aspects de grammaire ou de langage musical. Le livre de Célestin Deliège se présente à cet égard comme une source première.

Si Célestin Deliège est un témoin de son temps, il en est aussi une formidable mémoire. Car l'acuité et la solidité de son métier de musicologue se fondent, on le pressent à la lecture, mais on le sait avec certitude quand on a suivi son enseignement, sur une mémoire hors norme, c'est-à-dire sur une intériorisation de toute la musique et de tous les textes théoriques qui constituent les cinquante ans de modernité dont il parle. Ainsi, ces cinquante ans de modernité qu'entend cibler l'ouvrage correspondent-ils tout simplement, pour lui, aux cinquante ans que couvrent jusqu'à ce jour sa mémoire vive en tant que musicologue.

Configuration singulière et imposante, après ou à côté de laquelle il est difficile de se poser, surtout quand on est auteur d'un tout petit livre sur les liens entre musique et postmodernité. La postmodernité n'entre pas, a priori, dans le récit proposé, puisque celui-ci est consacré à la modernité, depuis Darmstadt jusqu'à l'Ircam. Mais la position de Célestin Deliège quant à la postmodernité est plus marquée que cela, puisque celui-ci précise dès l'avertissement : "l'auteur ignore si la postmodernité existe en soi». II évoque plus loin "l'épisode dénommé par ceux qui y croient "postmodernité"”, voyant en elle un des avatars, un "arrière-faix mal baptisé, de la modernité" (p. 648), défendant en cela, dans le plus neutre des cas, une position comparable à celle de Jean-François Lyotard dans sa première phase, position qui envisage le postmodernisme comme un modernisme à l'état naissant ${ }^{1}$.

Malgré cette polémique, il faut saluer dans les écrits antérieurs de Célestin Deliège, en ce qui concerne l'étude des différentes "stratégies de l'anamnèse", le dernier chapitre d'Invention musicale et idéologies (1986) qui se décline en trois points : "l'académisme", "faire coexister des langages" et "l'observation". Mais aussi, dans Cinquante ans de modernité musicale, une formule merveilleuse concernant un des parangons de la postmodernité dans ses premiers symptômes : "Le scherzo de Sinfonia [1968] est, en cette seconde moitié du siècle, un des rares moments où le concept fait voir l'image." (p. 494) N'est-ce pas, à cet égard, ce mouvement plutôt que l'opéra Votre Faust [1969] d'Henri Pousseur qui "a contribué à créer une religion de la citation", comme on peut le lire (p. 370)? La raison semble en être justement la suivante, Berio utilise la citation comme une Traumdeutung et avec virtuosité, comme un prestidigitateur éblouissant, alors que Pousseur se lance dans une démonstration didactique qui le conduit à tisser, en musique, une sorte d'histoire expresse, conduisant de Monteverdi à Webern, en un aller et même retour, puis, en une seconde étape, de Webern au bruit blanc et réciproquement. Berio est par là entré dans l'histoire et le répertoire, Pousseur non, me semble-t-il. Le procédé non hiérarchisé est plus séduisant que l'autre, n'est-ce pas? Et Berio est vraiment très fort pour lancer l'auditeur dans une aventure entre ancrage et inouï, entre champs de ruines et champs de forces, où l'anamnèse, la mémoire longue et la mémoire courte, le faire-mémoire sont à l'œuvre manifestement dans la composition musicale. 
Sur cette question de postmodernité, un second et dernier point. Il faut rappeler une évidence qui me semble tout à la fois affirmée et infirmée, dite et niée dans la somme de Célestin Deliège. L'Ircam, ici présenté comme le point d'aboutissement du récit, n'apparaît plus aujourd'hui, ni depuis plus de 10 ans, comme une institution monolithique. L'Ircam n'est plus le lieu exclusif de l'héritage post-wébernien. On y programme des musiques de tous bords, minimalistes, post-minimalistes. On y forme aussi des compositeurs se réclamant d'esthétiques diverses, de musique savante, mais aussi de musiques destinées aux médias de tout type. Célestin Deliège parle d'ailleurs de "pluralisme" (p. 962) — qu'il associe surtout à l'action de Laurent Bayle puis de Peter Szendy - de politique multi-culturelle, précisant : "L'accent est mis sur la diffusion, la communication, et la réception des compositeurs. " II rappelle la présence historique d'une certaine "dissidence" et l'implication de plus en plus marquée des multimédias, la collaboration avec les festivals, les scènes lyriques, la chorégraphie, le cinéma... (p. 956) L'aboutissement de cela peut facilement être constaté aujourd'hui. Or, Célestin Deliège écrit (p. 963) : "Comment retrouver des médiations sans retomber dans le lieu commun? On n'en accroît pas l'originalité en le nommant "postmoderne"..." Certes; cependant, on retrouve là décrits un certain nombre des symptômes qui le désignent, l'hybridité, l'esthésico-centrisme (pour reprendre une expression de Jean-Jacques Nattiez), l'attachement à un ancrage sémantique qui se dit aussi dans les liens avec le film ou la danse... L'Ircam est devenu quelque chose de tentaculaire, de multiple, ce qui justement infirme ou oblitère l'idée même d'une trajectoire qui serait unique, d'une évolution historique au singulier. Faut-il pour cela en conclure, comme on peut le lire encore (p. 963), "Parier à partir de ces éléments sur l'action future de l'Ircam est, en 2000, hasardeux"? L'action, mais à quelle fin? Là est la question.

\section{"L'évolution historique et ses marges", que faut-il entendre par ce titre?}

Le concept d'évolution historique est prioritaire pour Célestin Deliège. Ce concept évoque les enjeux majeurs auxquels il entend s'intéresser. II est rapporté à "la recherche musicale", à "la recherche sur le code", au "programme de recherche", aux "efforts de recherche de langage" (p. 308, à propos de Dutilleux), pour citer quelques expressions synonymes. L'idée d'évolution historique sous-entend une directionnalité du temps, un dévoilement du temps par une invention continuelle d'inouï, c'est l'idée même de modernité. Ecrire l'histoire revient alors à établir un "recensement de théories intentionnelles, prescriptives, et de poétiques nouvelles", selon le programme que Célestin Deliège définit lui-même (p. 54), isolant des causes et des effets.

On trouve une définition négative de ce qu'est "l'évolution historique" dans la manière dont Henri Dutilleux est présenté, peu de pages lui étant d'ailleurs consacrées. L'audience d'Henri Dutilleux, ainsi que la longévité de sa carrière créatrice sont essentiellement jugées à l'aune du "musicalement correct " américain. Célestin Deliège dit, pour commenter le succès que le compositeur français connaît aux États-Unis, "ce pays, où le répertoire contemporain est réfugiê dans les universités, n'a pas d'exigence particulière en matière d'évolution 
historique". Célestin Deliège dit ensuite, non sans humour, "le grand public peut accepter une modernité modérée, voire quelque peu pointue, quand elle est transmise par une vedette qui prend la place d'un agent de sécurité" (p. 307). L'évolution historique est donc une exigence que compositeur et public doivent servir.

Que faut-il alors entendre par l'autre terme de mon titre : ses "marges"? Mot bien peu musicologique, qui voudrait évoquer à la fois le lieu possible de la glose, des "tâches restantes" comme le disait François Nicolas dans l'argumentaire de la journée consacrée à l'ouvrage de Célestin Deliège. II s'agit pour moi de marginer, c'està-dire d'annoter en écrivant dans la marge de cette somme qu'est l'ouvrage de Deliège. Mais les "marges", voudraient évoquer aussi les compositeurs désignés comme ignorant la nécessité d'être acteurs de cette "évolution historique", les compositeurs qui sembleraient ne pas s'en mêler, qui se situeraient en dehors. Ceux qui se trouvent écartés de fait de cette histoire de la modernité, les marginaux.

Une dichotomie est donc présente, l'évolution historique et ses marges, dichotomie toujours latente, sous-jacente, posée et reposée par des comparaisons ou des exclusions, et donc, me semble-t-il, très présente pour le lecteur.

Ainsi, l'histoire est écrite ou décrite en termes de "responsabilité", "d'engagement", de "crédibilité". On lisait dêjà dans l'article "Le legs de 1912", "l'action de Schönberg a été sur ce point plus symptomatique que celle de Stravinsky, et sa responsabilité plus engagée ${ }^{2}$ ". Célestin Deliège parle d'un "devoir d'invention", et inventer c'est "rationaliser" (au sujet de Stravinsky), inventer "ce n'est pas l'éclectisme". Ou encore, de façon plus radicale, "il n'y a pas d'éclectisme en matière d'invention ${ }^{3}$ ". Déjà en 1985 , cela se cristallisait dans la formule "l'artiste, dans notre société du moins, doit être novateur, sans quoi son action est secondaire ${ }^{4}$. Ce devoir d'innover doit trouver en outre, pour être authentique selon Célestin Deliège, son appui sur ce qu'on pourrait nommer "un devoir de théorie", une implication grammaticale. La page 669 dit très clairement : "Ce livre est avant tout référé au concept." Paradoxalement, Célestin Deliège parle de "dommage causé à la musique par le projet cagien " (p. 139), projet qui me semble, quant à moi, bien souvent très conceptuel. Il faut donc entendre qu'il existe différentes sortes de concepts.

Selon cette logique qui privilégie la nécessité «d'avancer des concepts" (p. 669), Célestin Deliège affirme : "Le concept théorique a donc généralement précédé l'œuvre pendant la seconde moitié du xxe siècle." Or cette vérité peut presque généralement être contredite depuis le début des années 1970, même si elle a été très pertinente dans l'après-guerre, même si des exceptions notoires ont subsisté ensuite. Le dernier quart du siècle n'a-t-il pas été relativement pauvre en textes théoriques, en déclarations d'intention, cela allant de pair avec une incrédulité généralisée à l'égard des métarécits? Des compositeurs très actifs n'ont aucune production théorique, quelle que soit leur obédience. N'est-il pas symptomatique par exemple que les écrits de Ligeti, traduits et publiés en français en 2001, aient en réalité été écrits pour la plupart entre 1957 et 1970? Un texte, plus récent, de 1991, a été placé à la première place dans ce recueil, 
il est intitulé "Pensées rhapsodiques sur la musique en général et sur mes propres compositions en particulier". Ce texte est une sorte de manifeste, dans lequel Ligeti revendique une "modernité différente", entre, d'une part, une "syntaxe obligatoire", et d'autre part, un "postmodernisme" sous perfusion.

L'abondance d'écrits théoriques des années d'aprèsguerre $a$, on le sait, été généralement remplacée, dès les années 1970 et plus encore ensuite, par des entretiens ou de brefs textes informels, parcellaires et non systématiques, en tous les cas n'impliquant que rarement des déterminants techniques. On pourrait à cet égard évoquer le texte d'ouverture du premier numéro de la revue Entretemps, à l'automne 1986, dans lequel on peut lire : "Le rapport de la création au discours, à la théorie, est en train de s'inverser. Voici qu'il passe par une phase où réflexions critiques et formes écrites de la pensée musicale redeviennent plus nécessaires", et la tendance ne s'est pas inversée si vite. C'est donc bien là un témoignage, parmi d'autres, de compositeurs et de musicologues qui alors avaient une trentaine d'années à peine.

L'intrigue ne va-t-elle pas, parfois, dans Cinquante ans de modernité musicale, jusqu'à se nouer comme une utopie totalisante? Ainsi, parlant des années 1950-1960, Célestin Deliège évoque "un programme volontariste : la constitution d'un langage à travers le sérialisme", ce qui aurait évité, dit-il, le "désarroi" et les "failles déplorables" que nous connaissons aujourd'hui. II s'agit là d'un regard porté en arrière qui aimerait réécrire ou reconstruire les faits. Cependant, les postweberniens d'alors peuvent-ils absolument être qualifiés de "force centrifuge", capables de "tout rallier", y compris Stravinsky? Celui-ci écrivit effectivement avec des séries défectives après la mort de Schoenberg... cependant, le lecteur incrédule cherche les exceptions à ce "tout" de "tout rallier"... et trouve... au hasard, Poulenc (mort en 1963). Mais Poulenc existe-t-il vraiment? Pas dans l'index en tout cas. II n'existe pas dans le droit chemin tracé de Darmstadt à l'Ircam. Poulenc est-il néo-classique, pire, académique? Mais alors les postweberniens n'ont rallié que le tout d'une certaine modernité et non toute l'énergie compositrice, composante des années 1950.

Mais je suis là dans un cas extrême, à la marge des marges, on pourrait dire dans un cas banni par définition.

Que se passe-t-il dans une zone plus proche et cependant exclue du parcours? Célestin Deliège parle dans certains cas de "rectification", de "restauration et de répulsion", de "régression", entre autres dans un cas précis (p. 693) de régression "à l'anglaise" pour George Benjamin, ou encore à propos du même compositeur anglais de "défaillance acceptée" de la modernité européenne (p. 961).

Dans un autre domaine, des pans de modernité qui n'ont trouvé leur fondation ni à Darmstadt, ni à l'Ircam, se trouvent aussi éloignés de ce parcours de la modernité. Ainsi, pour Célestin Deliège, "Le minimalisme paraît avoir déjà vécu" (p. 648); cela n'est pas certain si l'on écoute des musiques post-minimalistes comme celles de John Adams ou de Louis Andriessen, il reste des traces indubitables de l'expérience et de l'énergie minimalistes (insupportable pour certains). Est-ce cependant une raison suffisante pour ne pas en parler? ou 
alors faudrait-il affirmer symétriquement que "le sérialisme aussi paraît avoir déjà vécu "? Son histoire n'estelle pas close? Mais "avoir vécu" signifierait alors simplement que minimalisme et sérialisme ont été promis à une mutation organique.

Toujours dans cette zone proche et cependant en dehors du parcours officiel, des compositeurs sont mentionnés comme des "parenthèses" (Aperghis par exemple, si présent dans les années 1960 comme compositeur de théâtre musical et très présent aussi dans la saison 2003-2004 de l'Ircam). D'autres le sont comme de valeureux chefs d'orchestre qui sauvent par leur baguette leurs œuvres dont la valeur authentique serait par ailleurs à démontrer. Il s'agit là de Peter Eötvös. D'autres sont déclarés ne pas devoir "être vraiment pris en charge" dans ce récit, il en va ainsi de Steve Reich. Ailleurs, Célestin Deliège parle de "compositeurs épuisés", de "compositeurs de tropes qui ont renoncé à être pionniers" et certes il en existe.

Je ne voudrais pas remplir les marges d'absents nommés dans le désordre, cependant le lecteur constate qu'il n'y a aucune trace de Maurice Ohana ni de Philippe Hersant - pour évoquer des générations différentes et des compositeurs cependant très prolixes. Chostakovitch n'est présenté qu'en deux phrases dans un "contrepoint" (p. 41) et nommé à nouveau brièvement au sujet de Wolfgang Rihm. On sait cependant que Chostakovitch a acquis du poids et de la présence le siècle avançant. Il est actuellement une référence pour de nombreux compositeurs.

II suffirait peut-être d'inscrire une seule question dans la marge. Est-il possible de parler, en tant que musicologue, d'une réalité musicale, d'une œuvre, qui ne soit pas systémique, dominante, univoque, sans pour autant que cette réalité musicale ne soit faible, sans acuité, sans rigueur, sans tension, bref, sans intérêt, sans idée musicale?

Je voudrais prendre un seul exemple, évoqué rapidement p. 675, celui de Trois sœurs de Peter Eötvös, opéra en trois séquences d'après la pièce de Tchékhov, créé en 1998 et dont la vie à la scène est réelle, puisqu'il a été redonné dans dix-neuf villes européennes, avec diverses mises en scène et dans diverses traductions ${ }^{5}$. Eötvös est reconnu pour son «invention à cent pour cent extra-musicale ${ }^{6}$, active même en dehors d'un dispositif théâtral et donc encore plus en présence de celui-ci. Mais cette invention extra-musicale fonde une efficacité magistrale dans le domaine du purement sonore. Eötvös sait dévoiler une part d'inouï, ce qui est aussi une définition de la modernité, même s'il joue conjointement sur des éléments d'ancrage dans un arrière-pays plus connu de ses auditeurs. L'opéra d'Eötvös n'est cependant pas écrit dans un style éclectique. Les seules "impuretés" sont une bande qui évoque les bruits de l'incendie de la petite ville de province où la scène se passe, des percussions de petites cuillères dans des tasses à thé et le sifflotement récurrent de Macha sur lequel s'achève l'opéra.

L'opéra s'ouvre par une réminiscence symbolique, l'âme russe flottant dans un accord tremblé d'accordéon. Pour Eötvös, "l'accordéon dans Trois sœurs, c'est vous, c'est moi, ce sont les cent ans qui nous séparent de leur histoire". C'est le pari de l'empathie suscitée par un décor sonore qui n'est pas désincarné. L'air d'accordéon qui 
s'échappe des cintres suggère que l'on est en Russie (malgré le décor japonais dans la mise en scène d'Amagatsu), mais dans une Russie mise à distance, car si l'accordéoniste est dans la fosse pour voir le chef, il se trouve dans une sorte de boîte en plexiglas. Le son transite par des haut-parleurs. L'accordéoniste a un rôle de mentor, à cause de cela, il est selon Eötvös "l'instrument hors histoire, extérieur à la situation scénique, ouvrant chacune des séquences ${ }^{7}$ ".

On l'entend en solo durant le prologue au début de la première séquence ainsi que dans le trio qui suit. II introduit à une sorte de douleur muette, entrecoupée de silences, figée sur deux intervalles (quinte juste et triton) clés pour l'ensemble de l'opéra, en accords tenus puis à la manière d'un air. Le texte du trio, "que la musique est gaie", chanté par les trois sœurs, reprend en fait les dernières répliques du drame de Tchékhov, ouvrant l'opéra mais nous projetant aussi dans le passé. Ce texte n'évoque donc pas la musique de l'accordéon, tout n'est que souvenir, car les trois sœurs qui chantent n'ont pas vu, ne verront pas leurs rêves de voyage et d'amour se réaliser. Tout est "compris" dans ce timbre et dans cette énergie particulière à l'accordéon.

On le retrouve au début de la deuxième séquence, l'accordéon est un des éléments des grands accords tenus par l'ensemble des forces instrumentales; puis au début de la troisième séquence où il maintient une sorte de bourdon en trémolo auquel se joignent les cordes et le bruit des petites cuillères, il participe au brouhaha des hommes qui prennent le thé.

L'accordéon devient une citation de timbre, pourquoi? Tout d'abord parce que l'accordéon par nature n'appartient pas à l'orchestre symphonique, pas plus que les petites cuillères des tasses à thé. II y a donc altérité. Ensuite, car il possède des qualités de condensation (il est chargé d'une histoire qu'il charrie et qui peut elle-même en charrier beaucoup d'autres), d'instantanéité (car il est perçu comme un élément déjà connu) et de globalité (par le fait même qu'il est un timbre, donc une sonorité complexe). II introduit à ce que Luciano Berio déjà désignait par la "possibilité d'une lecture utopique du monde ${ }^{8}$ " ouverte par la musique. Il s'agit, pour Eötvös, de continuer à poser pour notre siècle une utopie sonore faite d'énergie, d'inouï mais une utopie communicable, une utopie-empathie.

Il y aurait donc, j'ai voulu brièvement le signifier, une diversité de forces en présence dans la modernité de la seconde moitié du siècle dernier, qui ne peut sans doute se résoudre à une dichotomie, ni même se penser en mesurant des distances par rapport à un noyau dur ou à un trajet fléché.

On reste étonné du fait qu'il n'y ait pas de conclusion générale à cette étude. Le livre s'interrompt brusquement, à la verticale, avec l'évocation d'un cas particulier. Ceci est d'autant plus étonnant que l'œuvre est monumentale. La clé générale se trouve peut-être au tout début de l'ouvrage, dans le motif initial, c'est-à-dire dans l'exergue de Pierre Nora. "L'histoire contemporaine, cette exploration de l'actualité, ne consiste pas à appliquer au présent des méthodes historiques éprouvées pour le passé, elle est l'exorcisme ultime de l'événement, la dernière séquelle de sa résolution. Seraitelle contredite par l'histoire, il n'empêche qu'au même titre que l'événement, elle aura été. ${ }^{9}$ " Là se trouve pour 
moi le poids juste et la valeur unique du travail de Célestin Deliège tel qu'il nous a été donné de commencer à le lire.

\section{NOTES}

1. Lyotard, 1988 , p. 24.

2. Deliège, 1982, p. 185.

3. Deliège, 1982, p. 151.

4. Deliège, 1985, p. 38.

5. Si l'on se réfère à la recherche établie par Elisabetta Soldini pour L'Avant-Scène Opéra consacré à Trois sœurs, n² 204, p. 102-103.

6. Frank Langlois dans le catalogue des œuvres d'Eötvös aux éditions Salabert.

7. Trois sœurs. Programme de la création à l'Opéra de Lyon, le 13 mars 1998, p. 20.

8. Dalmonte, 1983, p. 37.
9. Pierre Nora, "Le retour de l'événement", dans Pierre Nora et Jacques Le Goff (dir.), Faire de I'histoire, tome 1, "Nouveaux problèmes", Paris, Gallimard, 1974, p. 210-218. (Note de la rédaction)

\section{BIBLIOGRAPHIE}

DALMONTE, R. (1983), Luciano Berio, entretiens avec Rossana Dalmonte, Paris, Lattès.

DELIÈGE, C. (1985), "Variables historiques du concept de recherche musicale", dans T. Machover (dir.), Quoi, quand, comment la recherche musicale, Paris, Bourgois, p. 35-60.

DELIÈGE, C. (1982), "Le legs de 1912", dans F. Lesure (dir.), Stravinsky: études et témoignages, Paris, Lattès, p. 149-192.

LIGETI, G. (2001), Neuf essais sur la musique, Genève, Contrechamps.

LYOTARD, J.-F. (1988), Le postmodernisme expliqué aux enfants, Paris, Galilée.

Ramaut-Chevassus, B. (1998), Musique et postmodernité, Paris, PuF, coll. "Que sais-je?". 